\title{
CLINICOHEMATOLOGICAL STUDY OF HEMOPHILIA PATIENTS IN BHOPAL
}

Rajendra Kumar Nigam¹, Rajni Choudhary², Reeni Malik ${ }^{3}$, Suhas Kothari ${ }^{4}$, Kaushal Prasad Verma ${ }^{5}$, Archana Shrivastava6, Neha Banseria7, Rubal Jain ${ }^{8}$

\section{HOW TO CITE THIS ARTICLE:}

Rajendra Kumar Nigam, Rajni Choudhary, Reeni Malik, Suhas Kothari, Kaushal Prasad Verma, Archana Shrivastava, Neha Banseria, Rubal Jain. "Clinicohematological Study of Hemophilia Patients in Bhopal". Journal of Evolution of Medical and Dental Sciences 2014; Vol. 3, Issue 11, March 17; Page: 2910-2916,

DOI: $10.14260 /$ jemds/2014/2224

ABSTRACT: INTRODUCTION: The X-linked inherited coagulation disorders, hemophilia A (Factor VIII deficiency) and hemophilia B (factor IX deficiency), together with Von Willebrand disease comprise 95 to 97 percent of all the Inherited deficiencies of coagulation factors. ${ }^{(1,2)}$ Replacement of the deficient factor is the mainstay of treatment; it may be "on demand" or "prophylactic" to prevent hemarthrosis in severe deficiency. The prevalence of hemophilia A is 1 in 5000 and that of hemophilia B is 1 in $30,000^{(3,4)}$ male live births and Von Willebrand disease with over all prevalence in the general population being 1:100.(4) Clinically both the deficiencies present as a lifelong bleeding disorder with considerable morbidity due to crippling arthropathy. Most common cause of death in these patients is Intra Cerebral Hemorrhage. Hemophilia A and B can only be distinguished on the basis of specific coagulation Factor VIII or IX assays. AIM: The present study was conducted with the aim to study the clinico-hematological profile of Persons With Hemophilia(PWH), their clinical presentations, incidence of inhibitors, estimating the burden of transfusion related complications at our hemophilia care center and to compare the findings with other studies of similar nature MATERIAL \& METHOD: This retrospective study was conducted in the Department of Pathology in collaboration with Hemophilia Care Centre at Gandhi Medical College, Bhopal during the period of August 2003 to July 2013. Diagnosis was made on the basis of history, physical examination and laboratory investigations such as bleeding time (BT), Prothrombin time (PT), Activated Partial Thromboplastin Time (APTT),correction studies(factor VIII and factor IX estimation whenever possible). CONCLUSION: Bleeding after injury is obvious in healthy people but difficult to decide, when it is due to bleeding disorder. Serious congenital conditions e. g. severe hemophilia becomes obvious in early childhood but may be misdiagnosed as non-accidental injury, whereas mild cases may go undetected till later years especially those who have not undergone surgery in early adult life. Presence of hemarthrosis, bruises, hematoma either spontaneous or traumatic in an otherwise normal child should warrants for investigating in the line of hemophilia. Repeated episodes early in life show severe hemophilia. Most common cause of death was found Intra Cerebral Hemorrhage (ICH).

KEYWORDS: Hemophilia, clinico-hematological-hemarthrosis, Bhopal.

INTRODUCTION: The X-linked inherited coagulation disorders, hemophilia A (Factor VIII deficiency) and hemophilia B (factor IX deficiency), together with Von Willebrand disease comprise 95 to 97 percent of all the inherited deficiencies of coagulation factors. ${ }^{1,2}$ Both factors take part in the intrinsic pathway of blood coagulation and affected individuals have severe, moderate and mild forms of disease, defined by factor plasma levels of $<1 \%, 2-5 \%$, and $6-40 \%$.respectively. The prevalence of hemophilia A is 1 in 5000 male live births and that of hemophilia B is 1 in 30,0003,4 and Von Willebrand disease with over all prevalence in the general population being 1:100.4 Clinically both 
the deficiencies present as a lifelong bleeding disorder with considerable morbidity due to crippling arthropathy. Hemophilia A and B can only be distinguished on the basis of specific coagulation Factor VIII or IX assays. ${ }^{5}$

Hemarthrosis is the most common, the most painful and the most physically, economically and psychologically debilitating manifestation of the hemophilia A.6, 7 Patient's susceptibility to musculoskeletal hemorrhage can lead to recurrent hemarthrosis and development of target joints. The definition of target joint is controversial but most accepted criterion is minimum of three bleeds into a single joint within a consecutive three month period. ${ }^{8}$

Replacement of the deficient factor is the mainstay of treatment however, if factor concentrates are either not available or not affordable, transfusion options including whole blood, FFP (fresh frozen plasma) \& Cryoprecipitate are still being used. Minor bleeds have also been controlled with antifibrinonlytic agents. ${ }^{9}$

The development of inhibitor is the most serious and challenging complication of hemophilia treatment with the enormous economic burden. Inhibitors are usually classified according to their levels in plasma as a "high titre" inhibitors, having activity $>5$ Bethesda units $(\mathrm{BU} / \mathrm{ml})$ or a "low titre" activity $<5 \mathrm{BU} / \mathrm{ml}$. In hemophilia an approximately $60-70 \%$ are high titre inhibitors and the remainder are low titre. Some patients develop transient inhibitors that never exceed a titre of 5 $\mathrm{BU} / \mathrm{ml}$ and disappear spontaneously with time.10

It is generally accepted that inhibitors screening should occur before invasive procedures and at regular intervals during the initial 50 treatment days as this is the highest risk period for inhibitor development. 11

AIM: The present study was conducted with the aim to study the clinico-hematological profile of PWH their clinical presentations, incidence of inhibitors, estimating the burden of transfusion related complications at our hemophilia care center. Further which type of clinical presentations must undergo coagulation profile testing to detect hemophilia cases at the earliest to decrease morbidity \& mortality. This can be helpful for policy makers to improve services to detect and treat these patients.

MATERIAL AND METHODS: This retrospective study was conducted in the Department of Pathology in collaboration with Hemophilia Care Centre at Gandhi Medical College, Bhopal during the period of August 2003 to July 2013.

A total 256 persons with coagulation disorder visiting for various laboratory tests with different medical and surgical complaints finally diagnosed as hemophilia were included in this study. A thorough history including family history, findings of physical examination \& laboratory tests were taken from medical records.

Diagnosis was made on the basis of history, physical examination and laboratory investigations such as bleeding time (BT), Prothrombin time (PT), Activated Partial Thromboplastin Time (APTT),correction studies and wherever possible specific coagulation factor assay. Correction or mixing studies using pooled normal plasma (PNP) was done to identify the prolongation of coagulation time due to factor deficiency or circulating anticoagulant inhibitors.

Other hematological investigations like complete blood counts including peripheral blood smear were also made to see blood cell morphology and platelet count \& blood grouping. Transfusion transmitted infections testing by ELISA were done as per the departmental standard operating procedures. Results of the investigations were recorded and analyzed. 
EXCLUSION CRITERION: Patients with platelet disorders were excluded from the study. Ethical clearance was taken from institutional ethical committee.

RESULTS: In this study 260 cases attending with history of prolonged bleeding along with various medical and surgical complaints, finally diagnosed as hemophilia \& Von Willebrand disease in the Department of Pathology GMC \& Hamidia hospital Bhopal were included. A total of 254 cases of hemophilia were found, out of which 224 (86.15\%) \& 30 (11.53\%) were hemophila A \& hemophilia B respectively, and $6(2.3 \%)$ were Von Willebrand disease. Further analysis of 254 hemophilia patients was done.

\begin{tabular}{|c|c|c|}
\hline Age (in years) & No. of Patients & Percentage \\
\hline $0-5$ & 80 & $31.50 \%$ \\
\hline $6-15$ & 102 & $40.16 \%$ \\
\hline $16-30$ & 56 & $22.05 \%$ \\
\hline$>30$ & 16 & $6.30 \%$ \\
\hline
\end{tabular}

\section{TABLE 1: AGE DISTRIBUTION OF THE HAEMOPHILIA PATIENTS (N=254)}

The predominant age group affected was between 6-15 years -102 cases $(40.16 \%)$ (Table 1$)$, although they were ranging from 6 months to 45 years.

\begin{tabular}{|l|c|c|c|c|c|c|}
\hline & \multicolumn{2}{|c|}{$\begin{array}{c}\text { Factor VIII } \\
\mathbf{2 2 4} \text { cases }\end{array}$} & \multicolumn{2}{c|}{$\begin{array}{c}\text { Factor IX } \\
\text { 30 cases }\end{array}$} & \multicolumn{2}{c|}{$\begin{array}{c}\text { Total } \\
\text { 254 cases }\end{array}$} \\
\hline \multicolumn{1}{|c|}{ Bleeding site } & No. & $\mathbf{\%}$ & No. & \% & No. & \% \\
\hline Joints (Haemorthrosis) & 148 & $66.07 \%$ & 17 & $56.67 \%$ & 165 & $64.96 \%$ \\
\hline Muscle & 40 & $17.85 \%$ & 10 & $33.34 \%$ & 50 & $19.68 \%$ \\
\hline Gum & 18 & $8.35 \%$ & 0 & 0 & 18 & $7.08 \%$ \\
\hline Petechiae & 8 & $3.54 \%$ & 3 & $10 \%$ & 11 & $4.33 \%$ \\
\hline Epistaxis & 10 & $4.46 \%$ & 0 & 0 & 10 & $3.97 \%$ \\
\hline
\end{tabular}

TABLE 2: $1^{\text {ST }}$ PRESENTATION OF HAEMOPHILIA PATIENTS

In our study most common presentation was joint bleeding-64.96\%, followed by muscle 19.68\% \&gum bleeding 7.08\%, petechiae 3.54\%, and epistaxis $4.46 \%$ (table 2).

In Von Willebrand disease manifestations are mainly in the form of mucocutaneous bleeding such as petechiae ( $4 / 6$ cases) and epistaxis ( $2 / 6$ cases).

\begin{tabular}{|c|c|c|c|c|c|c|}
\hline Disease & \multicolumn{2}{|c|}{ < episode / year } & \multicolumn{2}{c|}{ 1-5 episode / year } & 6-19 episode / year \\
\hline & No. & $\mathbf{\%}$ & No. & \% & No. & \% \\
\hline Hemophilia A n=224 & 130 & $51.18 \%$ & 60 & $23.62 \%$ & 34 & $13.38 \%$ \\
\hline Hemophilia B n=30 & 16 & $6.29 \%$ & 10 & $3.93 \%$ & 4 & $1.57 \%$ \\
TABLE 3: FREQUENCY OF BLEEDING EPISODE PER YEAR (N=254)
\end{tabular}

Out of 254patients, 146 (57.48\%) cases had at least one bleeding episode during the study. 108 (42.51\%) cases experienced two or more bleeding episode per year (Table 3). 


\begin{tabular}{|l|c|c|c|c|}
\hline & \multicolumn{2}{|c|}{$\begin{array}{c}\text { Hemophilia A } \\
\text { N=224 }\end{array}$} & \multicolumn{2}{c|}{$\begin{array}{c}\text { Hemophilia B } \\
\text { N=30 }\end{array}$} \\
\hline & No. & \% & No. & \% \\
\hline Spontaneous bleeding & 128 & $57.14 \%$ & 16 & $53.33 \%$ \\
\hline Bleeding following trauma / surgery & 96 & $42.85 \%$ & 14 & $46.66 \%$ \\
\hline
\end{tabular}

TABLE 4: TYPES OF BLEEDING AMONG THE HEMOPHILIC PATIENTS (N=254)

In $110 / 254$ (43.30\%) patients, the bleeds were trauma induced whereas the majority of patients experienced spontaneous bleeds 144/254 (56.69\%) (Table 4).

\begin{tabular}{|l|c|c|c|c|}
\hline \multirow{3}{*}{ Severity } & \multicolumn{2}{|c|}{$\begin{array}{c}\text { Hemophilia A } \\
\text { N=224 }\end{array}$} & \multicolumn{2}{c|}{$\begin{array}{c}\text { Hemophilia B } \\
\text { N=30 }\end{array}$} \\
\cline { 2 - 5 } & No. & \% & No. & \% \\
\hline Mild & 64 & $25.19 \%$ & 18 & $60 \%$ \\
\hline Moderate & 87 & $38.84 \%$ & 08 & $26.67 \%$ \\
\hline Severe & 76 & $33.93 \%$ & 04 & $13.33 \%$ \\
\hline \multicolumn{3}{|c|}{ TABLE N0. 5 SEVERITY OF HEMOPHILIA (N=254) }
\end{tabular}

Majority were in moderate group in hemophilia A \& mild group in hemophilia B. On the basis of clinical features important being number of episodes and type of bleed e.g. ICH always to be considered as severe factor deficiency. Patients of hemophilia A and B were categorized as 61/224(27.23\%) and $18 / 30(60 \%)$ mild hemophilia, 87/224 (38.84\%) \& 8/30 (26.67\%) moderate hemophilia and 76/224(33.92\%) \& 4/30 (13.33\%) severe hemophilia respectively.

Complete blood count was found almost normal in all except 44 (17.32\%) patients having frequent bleeding episode who had raised platelet count. Results of coagulation screening tests showed that $100 \%$ patients of hemophilia had prolonged APTT, although range was from 43 to 68 seconds in comparison to 30 seconds in control.

In our series HBsAg \& HCV seropositivity was found only in 2 \& 4 cases where as no patient found HIV positive. Although only 80 patients participated in screening for TTIs (transfusion transmitted infections), although blood/blood products are used in our patients whenever factor concentrates are not available or not affordable, for this low incidence of TTI, credit can be given certainly to good blood bank practices.

We have found inhibitors in $9.09 \%$ ( 4 cases out of 44 cases studied, not all cases were tested for inhibitors due to non-availability of consent)

Most common cause of death in our series was ICH(Intra Cerebral Hemorrhage). $>50 \%$ (.in $7 / 13$ cases overall) and all 7 were of hemophilia A.

DISCUSSION: The age group in our study ranged from 0-45 years of age compared to less than 15 years by Uddin MM \& Karim et al ${ }^{12,13}$ \& less than 18 years of age by MM Ha et al. ${ }^{14}$ All the cases were male as in other studies. Presenting symptoms were most commonly hemarthrosis (64.96\%) similar to Uddin MM et al (100\%) \& Karim et al (82\%) while MM Hazewinkel ${ }^{12-14}$ reported subcutaneous bleed(45\%) as most common symptom. 
Other common symptom in decreasing order of frequency were muscle bleed(19.68\%), gum bleed (7.08\%), skin bleed (4.33\%), epistaxis (3.97\%) while Uddin MM12 reported wound bleeding (52\%) \& bleeding after tooth extraction (38\%), Karim et al ${ }^{13}$ reported gum bleeding (38\%) \& bleed during surgical procedure such as circumcision \& tooth extraction (28\%) other rare presentations were hematemesis, malena, epistaxis (2\%) while mucosal bleeding (15\%) was reported by $\mathrm{M} \mathrm{H}$ Hazewinkel. ${ }^{14}$

Age at first presentation in our study was less than 5 years in $55.1 \%$ cases $(29.98 \%$ were less than 1 year), while Karim MA et al ${ }^{13}$ reported $94 \%$ cases in less than 5 years age group (64\% were less than 1 years). No cases were reported in neonatal period by any study including ours. We found positive family history in $70 \%$ of cases same as by Karim et al, while M H Hazewinkel \& Uddin MM reported $49 \%$ \& $30 \%$ respectively. ${ }^{13-15}$

We have found mild disease in $25.19 \%$ \& 60\% of hemophilia A \& B, moderate disease in $38.84 \%$ \& $26.67 \%$ in hemophilia A \& B, severe disease in $33.93 \%$ \& $13.33 \%$ in hemophilia A \& B, while MM Uddin et al ${ }^{12}$ reported $45 \%, 42.5 \%$ \& $12.5 \%$ as mild, moderate \& severe respectively. Rahman M 15 found 45.5\% each in mild \& moderate \& 9\% in severe group. Rodgers reported 20\%, $30 \% \& 50 \%$ as mild moderate \& severe. Karim ${ }^{13}$ reported $52.5 \%$, \& $40 \%$ as mild disease in Hemophilia A \& B, moderate-47.5 \% \& 50\% in Hemophilia A \& B and $10 \%$ in severe hemophilia B ,no case has been found in Hemophilia A. M H Hazewinkel ${ }^{14}$ found $22 \%, 29 \%$ \& 43\% in mild, moderate \& severe respectively. These variations may be due to difference in population studied, health care facilities available \& social paradigms. Most important laboratory diagnostic tool was increase in APTT which was found raised in $100 \%$ of our hemophilic patients, while same had been found in majority of cases by others workers. Spontaneous bleed was reported in $57.14 \%$ \& $53.33 \%$ cases of hemophilia A \& hemophilia B by us, compared to $100 \%$ in severe groups $\& 17.5 \%$ in moderate groups by Uddin MM et al. ${ }^{12}$

Confusion may arise when hemarthrosis is not the presenting problem as in our study $35 \%$ of cases first presented with muscle \& gum bleed, petechiae \& epistaxis, also marked bruising, ecchymosis and epistaxis was the first presenting feature rather than hemarthosis was reported by HL Minhas et al 2014, Morgan LM et al 1993, \& Ljung R et al 1990. Primary cause of delayed diagnosis is failure among physicians to recognize the disease when presented with the clinical features more so when not with hemarthrosis.

CONCLUSION: It is the frequency \& persistence of blood loss together with minimal trauma, specially presence of hemarthrosis, bruises, hematoma either spontaneous or traumatic in an otherwise normal child should alert the physician to investigate for hemophilia even in the absence of family history. Early recognition is important to establish correct treatment and to avoid unnecessary investigations. Prolonged APTT with normal PT \& other coagulation tests favors hemophilia, type of which can be confirmed by mixing studies / specific factor assay. Repeated episodes early in life show severe hemophilia. Most common presenting symptom found was hemarthrosis $(64.96 \%)$ and cause of death was ICH ( $>50 \%)$.

Most of the centers in developing countries including India do not have facility for factor concentration estimation, hence clinically we can assess severity of hemophilia with type of bleed, like ICH is always to be considered as severe for treatment purposes to save the life. Factor replacement is the only treatment for hemophilia, ideally recombinant one that too preferably prophylactic. The specialty of transfusion medicine can be a core part of hemophilia care by providing 
the laboratory services in the form of hemostasis \& serology testing, testing for inhibitors, factor concentrates \& blood component support. Thus, we can achieve PWH (Persons with Hemophilia) without pain \& disability.

We would like to extend special acknowledgement to Prof. Dr. V.K. Bharadwaj, Dr. U. M. Sharma, HFI, Hemophilia Society Bhopal.

\begin{tabular}{|c|l|}
\hline FFP & Fresh Frozen Plasma \\
\hline VWD & Von Willebrand disease \\
\hline PWH & Persons with Hemophilia \\
\hline BT & Bleeding time \\
\hline PT & Prothrombin time \\
\hline APTT & Activated Partial Thromboplastin Time \\
\hline PNP & Pooled normal plasma \\
\hline ELISA & Enzyme linked immunosorbent assay \\
\hline TTD & Transfusion transmitted disease \\
\hline TTI & Transfusion transmitted infection \\
\hline HBsAg & Hepatitis B surface antigen \\
\hline HCV & Hepatitis C virus antibody \\
\hline HIV & Human immunodeficiency virus antibody \\
\hline ICH & Intra-cranial hemorrhage \\
& ABBREVIATIONS \\
\hline
\end{tabular}

\section{REFERENCES:}

1. Mannucci PM, Tuddenham EG. The hemophilias-from royal genes to gene therap. N Engl J Med 2001:344:1773-1779.

2. Mannucci PM. Hemophilia: Treatment options in the twenty first century - J Thromb Haemost 2003; 1:1349.

3. Bolton - Maggs P H, Pasi KJ. Hemophilias A and B. Lancet 2003, 361: 1801 - 1809.

4. Tejinder Singh. Atlas and text of Hematology. Second edition: Aug 2011, pp -309.

5. Roberts HR, Escobar M, White GC. Hemophilia A and B. In: Lichtman MA, Beutler E, Kipps TJ, Seligson U, Kaushansky J, Prchal JT, Williams Hematology 7th edition. New York: McGraw Hill; 2006 p-1867-86.

6. Roberts HR. Coagulation disorders, in: Williams Hematology, 5th edition. Baltimore: Wilkins, 1995.pp-1413-36.

7. Gaffney PJ, Thomas DP. Haemostasis and Thrombosis. Am J Clin Path 1997; 69:299, 359.

8. Blanchette VS, McCready M, Achonu C, Abdolell M, Rivard G, Manco - Jonson MJ. A survey of factor prophylaxis in, boys with hemophilia followed in North American hemophilia treatment centers. Hemophilia, 2003;9;19-26.

9. Marwaha N. Transfusion related complication in hemophilia. Asian J Transfus Sci 2013;7:6-7.

10. Tarek M, Owaidah (2012).Hemophilia inhibitors prevalence, causes and diagnosis, Hemophilia, Dr Angelika Batorova (Ed).ISBN:978-953-51-0429-2. 
11. Dubey A, Verma A, Elhence P, Agarwal P. Evaluation of transfusion -related in complication along with estimation of inhibitors in patients with hemophilia, A pilot study from a single center. Asian J. Transfus Sci 2013:7:8-10.

12. MM Uddin, Rahman MJ, Rahman MM, Sultana SA, Shah MS. Clinico-pathological study on haemophilia: An Analysis of 50 cases. J Bangladesh College of Physicians \& Surgeons, Vol 24, No.2, May 2006.

13. Karim MA, Siddique R, Jamalcy, Islam A. Clinical profile of haemophilia in children in A Tertiary care hospital. Bangladesh J Child Health 2013: vol 37(2); 90-96.

14. MH Hazewinkel, JJ Hoogerwart, P B Hesseling, P Hartley, PG Maclean, M Peters, G Wessels. Hemophilia patients aged 0-18 years in the western Cape. October 2003, Vol 93, No.93.10

15. J J Hoogerwerf, PB Hesseling, P Hartley, PE Maclean, M Peters, G Wessels. Haemophilia patents aged 0-18 years in the western cape. Oct 2003, vol 93, No, 10 SAMJ.

16. Rahman M. Clinico-pathology study on haemorrhagic disorder (dissertation).Dhaka, Bangladesh college of physicians and surgeon, 1998.

17. Minhas HL, Giangrande PLF. Presentation of severe haemophilia -A role of accident and emergency doctors -Feb -12, 2014 -Published by group.bmj.com.

18. Morgan LM, Kissoon N, deVebber BL. Experience with the hemophilic child in pediatric emergency department. Emerg Med 1993;11;519-24.

19. Ljung R, Petrini P, Nilsson. Diagnostic symptoms of severe and moderate haemophilia A and B. Acta Pediatr Scand 1990;79;196-200.

\section{AUTHORS:}

1. Rajendhra Kumar Nigam

2. Rajini Choudhary

3. Reeni Malik

4. Suhas Kothari

5. Kaushal Prasad Verma

6. Archana Shrivastava

7. Neha Banseria

8. Rubal Jain

\section{PARTICULARS OF CONTRIBUTORS:}

1. Professor, Department of Pathology, Gandhi Medical College, Bhopal, Madhya Pradesh.

2. Assistant Professor, Department of Pathology, Gandhi Medical College, Bhopal, Madhya Pradesh.

3. Professor and Head of the Department, Department of Pathology, Gandhi Medical College, Bhopal, Madhya Pradesh.

4. Associate Professor, Department of Pathology, Gandhi Medical College, Bhopal, Madhya Pradesh.
5. Senior Laboratory Technician, Department of Pathology, Gandhi Medical College, Bhopal, Madhya Pradesh.

6. Assistant Professor, Department of Pathology, Gandhi Medical College, Bhopal, Madhya Pradesh.

7. PG Student, Department of Pathology, Gandhi Medical College, Bhopal, Madhya Pradesh.

8. PG Student, Department of Pathology, Gandhi Medical College, Bhopal, Madhya Pradesh.

\section{NAME ADDRESS EMAIL ID OF THE CORRESPONDING AUTHOR:}

Dr. Rajendra Kumar Nigam,

C-116, Shahpura,

Bhopal, Madhya Pradesh,

India - 462039.

E-mail: dr.rajendranigam@gmail.com

Date of Submission: 19/02/2014.

Date of Peer Review: 20/02/2014.

Date of Acceptance: 03/03/2014.

Date of Publishing: 14/03/2014. 